\title{
THE EXPERIMENTAL EVOLUTION OF AGING IN FRUITFLIES
}

\author{
Stephen C. Stearns, Martin Ackermann and Michael Doebeli \\ Zoology Institute, University of Basel, Rheinsprung 9, CH-4051 Basel, Switzerland
}

\begin{abstract}
The evolutionary theory of aging suggests that the level of repair will evolve to an intermediate optimum that permits the accumulation of random damage to cells. This, in turn, causes a decline in essential functions during the life span of an organism. The central claim of the life history theory of aging is that intrinsic mortality rates evolve in response to changes in extrinsic mortality rates. To prove this central claim, it must be evaluated experimentally. Experimental evolution is an approach that has been yielding interesting results from both a variety of questions posed and organisms examined. In this article the organism chosen for study is the fruitfly (Drosophilia melanogaster) in which the evolutionary effects of high and low adult mortality rates are compared. It has been found that higher extrinsic mortality rates lead to the evolution of higher intrinsic mortality rates and a shorter life span. This is the first clear experimental demonstration of the central claim of the evolutionary theory of aging. () 1998 Elsevier Science Inc.
\end{abstract}

Key Words: evolution, aging, Drosophila

\section{INTRODUCTION}

AgING Is part of the evolution of the whole life cycle, from birth to reproduction to death, which is studied in the part of evolutionary biology called life history evolution (Roff 1992; Stearns 1992). Life histories result from the interaction of extrinsic mortality rates with intrinsic tradeoffs, such as maintenance vs. reproduction. Trade-offs imply that a change in one trait that increases fitness causes changes in other traits that decrease fitness. Because the classic life history trade-off between reproduction and survival is directly involved in the evolution of life span and intrinsic mortality rates, life history theory is that part of evolutionary biology most directly concerned with the prediction of aging.

In this article I will briefly review the evolution of life span and mortality rates, connect these rates to the molecular and evolutionary genetic approaches to aging, and attempt to illustrate how one can use the life history approach in an evolutionary experiment to test a central prediction of the evolutionary theory of aging, i.e., the evolution of intrinsic adult mortality rates

Correspondence to: Stephen C. Stearns. Tel: +41-61-267-3585; Fax: +41-61-267-3457; E-mail: stearns@ubaclu. unibas.ch 
is driven by changes in extrinsic adult mortality rates. The prediction-the higher the extrinsic mortality, the higher the intrinsic mortality— has been confirmed experimentally.

\section{Life history evolution of optimal life span}

Reproductive life span evolved as a balance between the costs and the benefits of increasing the number of reproductive events per lifetime. The costs are thought to be paid primarily through trade-offs between reproductive performance late in life and reproductive performance early in life. Age-specific selection pressures adjust the length of life to an intermediate optimum determined by the interaction of mortality rates with trade-offs. It would, thus, be optimal that mortality rates would increase and fecundity rates would decrease after a certain age. The life history definition of aging (Partridge an Barton, 1993) includes, but is more comprehensive than, a simple decrease in survival rates or reproductive output with age. Aging is defined as a decrease in the product, i.e., in the expected number of offspring per age class - with age. It, thus, summarizes the internal degradation of the organism as a decrease in reproductive performance, taking into account both survival and reproduction.

Even if there was no genetic variation among individuals within a population for age-specific reproductive and mortality rates (an unlikely assumption), life span could have been adjusted by past selection to an optimal value determined by phylogenetically inherited trade-offs. If this were correct, species would be characterized by a fixed, species-specific optimal life span and one could speak of a genetically programmed, fixed life span. However, in fruitflies and humans, this is not the case, for considerable genetic variation for life span is present, and only the broad limits on possible life histories are set by trade-offs that characterize the whole species.

Given that a certain trade-off structure has evolved, and that genetic variation is present that can make possible a response to selection, can selection pressures lengthen life? Any factor that decreases the reproductive contributions of juveniles and younger adults or that increases the reproductive contributions of older adults will lead to the evolution of a longer life. Furthermore, lower extrinsic mortality rates in the older age classes and higher extrinsic mortality rates in the younger age classes, or increased variation in extrinsic mortality rates in the younger age classes and decreased variation in extrinsic mortality rates in the older age classes, will likewise lead to the evolution of longer life. Such variations in mortality rates can also have important effects on fitness.

If an optimal life span has evolved, the mechanisms causing the trade-offs that determine the optimal life span may include a contribution from the genetic effects-antagonistic pleiotropy and mutation accumulations - that Medawar (1952) and Williams (1957) identified as causes of aging. However, those effects can increase mortality rates and decrease fecundity rates late in life beyond the levels predicted from optimal allocation. Life history theory assumes trade-offs based on population means, and does not take genetic variation within populations into account. A phenotypic theory based on optimization procedures assuming fixed trade-offs cannot explain or account for all the effects of aging, for they include a contribution from genetic mechanisms that are not fully represented in the theory. Although part of aging is adaptive and can be thought of as an optimal outcome, other aspects of aging are certainly maladaptive.

Organisms that reproduce sexually have a clear separation of germ line from soma, and return from a multi-to a single-cell stage at some point in their life cycle and cannot avoid aging. For organisms that reproduce by simple, perfectly symmetrical division, the force of selection does not decrease with the age of the individual, and the reproductive life span should be potentially 
unlimited. However, asymmetrical division, which results in a mother (older) and a daughter (younger), may also be a sufficient condition for aging to evolve (Partridge and Barton, 1993). If so, yeast and some bacteria may senesce and could provide good model systems for the experimental evolution of aging.

\section{The evolution of aging: genetic mechanisms}

The genetic explanation of aging is represented by at least two very different views (Rose, 1991). The first is the molecular approach, which concentrates on mechanisms shared by all living things. It views aging organisms as machines with parts that malfunction and assumes that the machine will run longer if the parts are fixed by manipulating a few genes that have large effects. The second is the evolutionary genetics approach, which views aging as a byproduct of selection for reproductive performance. Aging arises through the accumulation of many genes that have small positive or neutral effects on fitness components early in life, and negative effects on fitness components late in life. If the effects early in life are positive, they contribute to antagonistic pleiotropy (Williams, 1957). If these are neutral, they contribute to mutation accumulation (Medawar, 1952). Evolutionary geneticists, who believe that aging results from so many defects of such diverse origin that every time one of them is fixed another will immediately manifest itself, are skeptical of therapies for aging based on single-gene interventions.

\section{Age-specific selection on mortality rates}

According to Medawar (1952), "the force of natural selection weakens with increasing age-even in a theoretically immortal population, provided only that it is exposed to real hazards of mortality. If a genetic disaster ... happens late enough in individual life, its consequences may be completely unimportant." Methods for calculating the force of natural selection on different age classes were later worked out by Hamilton (1966) and Charlesworth (1980), who confirmed in detail what Medawar had asserted in general: the older the organism, the less it contributes to future generations, and the weaker the force of selection upon it. This key evolutionary insight may explain the evolution of aging through any of a variety of genetic, molecular, and physiological mechanisms, and it has become a major component of the life history theory.

\section{Artificial selection experiments on life span}

More than 14 artificial selection experiments designed to measure the trade-offs involved in aging have been conducted with the fruitfly, Drosophila melanogaster. The basic design is to select only one trait, such as late fecundity or life span, long enough to produce a significant response to selection. The correlated response in other traits, such as early fecundity or age at maturity, is then measured. The results suggest that early and late fitness components do trade off, but whether life span trades off with early fecundity or with age and size at maturity depends on how and in which laboratory the flies were selected.

\section{The disposable soma theory}

Because the molecular and evolutionary views observe a single reality, they must converge at some level. The most convincing attempt to combine them is Kirkwood's (1985) disposable soma theory, which states that the optimal level of investment for repair will be below the level required for indefinite survival because investment in maintenance trades off with investment in 
reproductive performance early in life. According to this theory, better maintenance is possible and is present in the germ line, it has just not been selected in the soma. This has focused attention on molecular repair mechanisms. Are repair mechanisms in the germ line more efficient than repair mechanisms in the soma? If one transplants them into the soma, do they rescue the soma and eliminate aging as an outcome? That would strongly confirm Kirkwood's (1985) theory.

\section{Experimental evolution and our experiment}

In contrast to artificial selection experiments, in which the researcher determines the trait selected, in experimental evolution the researcher poses a problem and lets the population determine the solution. This approach has recently been applied to bacteria, single-celled algae, and guppies with interesting results. In an ongoing experiment on fruit flies since November 1993, the problem we posed is defined by differences in adult mortality rates. One treatment involves the induction of high adult mortality rates (HAM treatment). The other treatment involves the induction flow adult mortality rates (LAM treatment). In both treatments the densities of larvae an adults are exactly the same. This is the first experiment in which adult mortalities have been varied while density has been held constant. The main questions asked by this experiment are these: do intrinsic mortality rates evolve as expected in response to differences in extrinsic mortality rates? That is, do the LAM flies evolve lower intrinsic mortality rates and longer life spans than the HAM flies? Are the optimality models of life history theory qualitatively confirmed? Is there compensating evolution that reduces the costs caused by trade-offs? Do the trade-offs themselves evolve?

\section{MATERIALS AND METHODS}

The base stock was derived from 43 isofemale lines collected near Basel, Switzerland, and crossed in large population cages several months before the treatments were initiated. There are two adult mortality treatments with three replicate population cages per treatment. All flies experience the same larval and adult density. In the treatment with high adult mortality rates (HAM), 90\% of the flies in the cage are killed and replaced twice per week; the probability of surviving one week as an adult is 0.01 . In the treatment with low adult mortality rates (LAM), $20 \%$ of the flies in the cage are killed and replaced twice per week; the probability of surviving one week as an adult is 0.64 . Since January 1996, this has been dropped to $10 \%$ mortality per observation or $81 \%$ survival per week.

\section{THEORETICAL PREDICTIONS}

Given that shorter development time can only be achieved with a smaller size when the flies eclose (hatch out of the pupae), that fecundity depends on size at eclosion and that, for a given size, life time fecundity is fixed, life history theory predicts that HAM females will hatch $52 \mathrm{~h}$ earlier and have higher fecundity early in life and lower fecundity later in life than LAM females. The fecundity curves should cross about $140 \mathrm{~h}$ (5.8 days) after the HAM files hatch (Fig. 1). Given that intrinsic mortality increases with fecundity and that intrinsic mortality conforms to Gompertz functions, which increases with age, life history theory also predicts that intrinsic mortality should, at evolutionary equilibrium, be higher in HAM than in LAM, and that this difference should increase with age. Thus, the life span of HAM flies should evolve to be shorter than the lifespan of LAM flies (Fig. 2). 


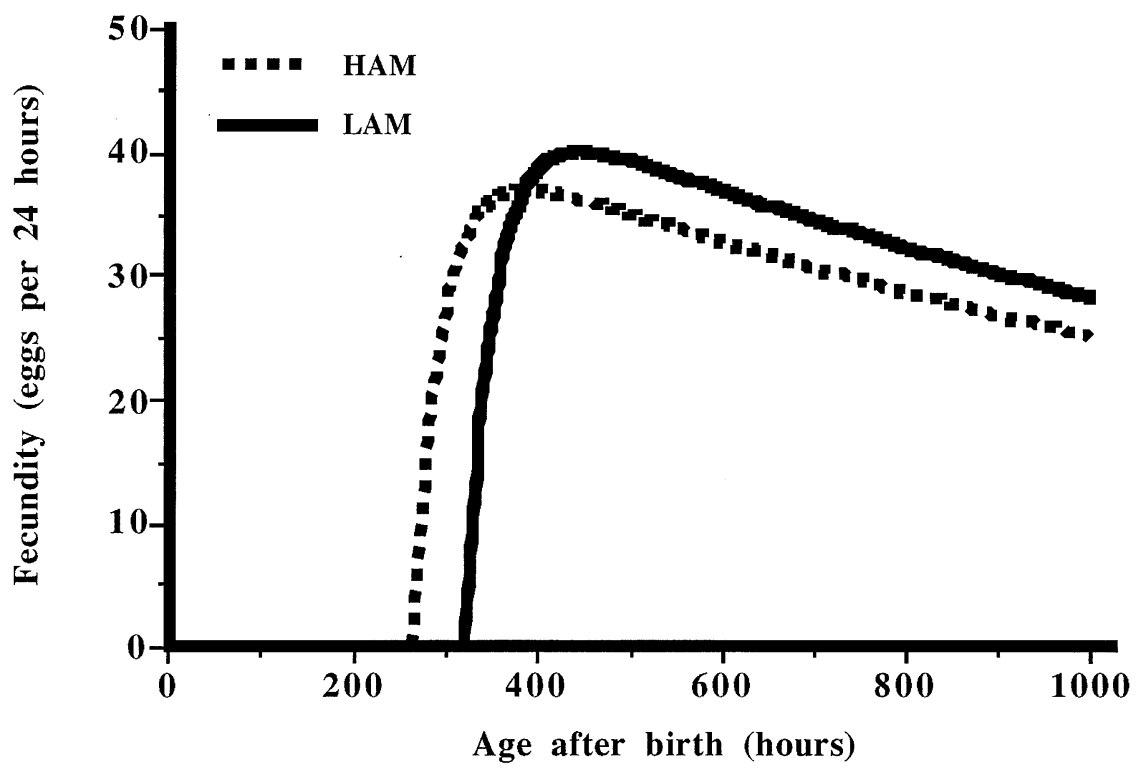

FIG. 1. Theoretical prediction of the optimal fecundity curves at evolutionary equilibrium based on two assumptions: flies that take longer to develop reach a larger adult size, and flies of larger adult size can lay more eggs.

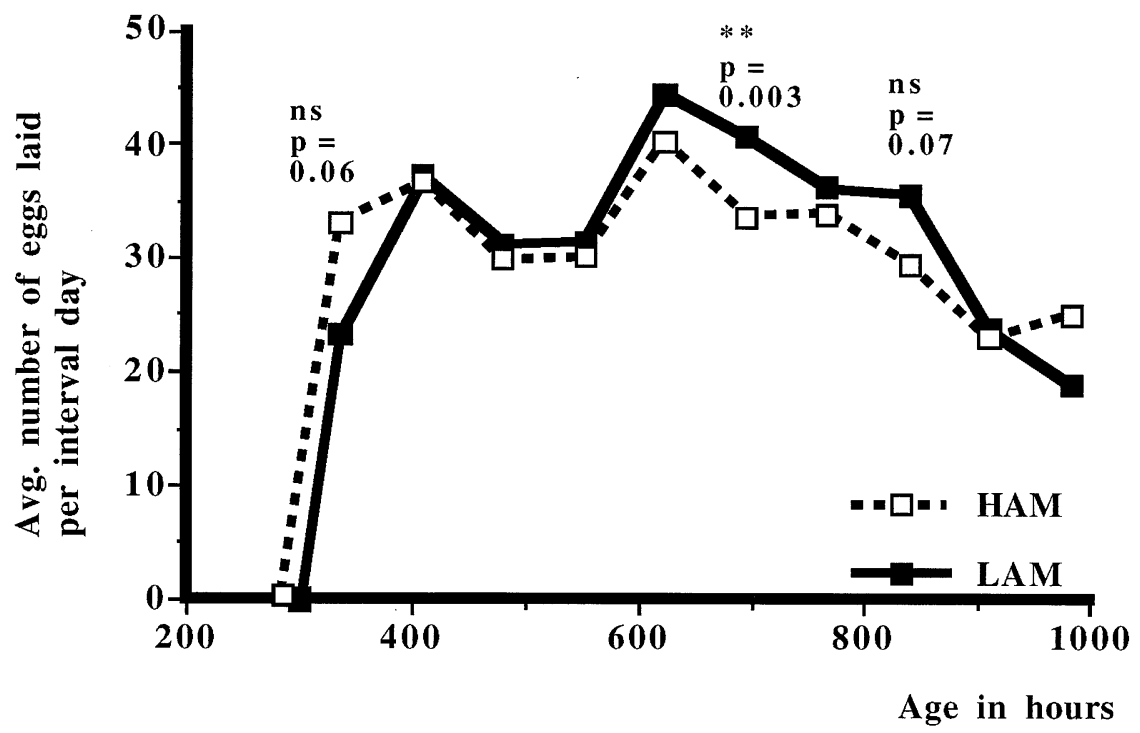

FIG. 2. The actual fecundity curves measured in 1996 after 2.5 years of experimental evolution. Flies that have encountered consistently high adult mortality (HAM) develop faster, hatch earlier at a smaller size, but lay more eggs early in life than do flies that have encountered consistently low adult mortality (LAM). 


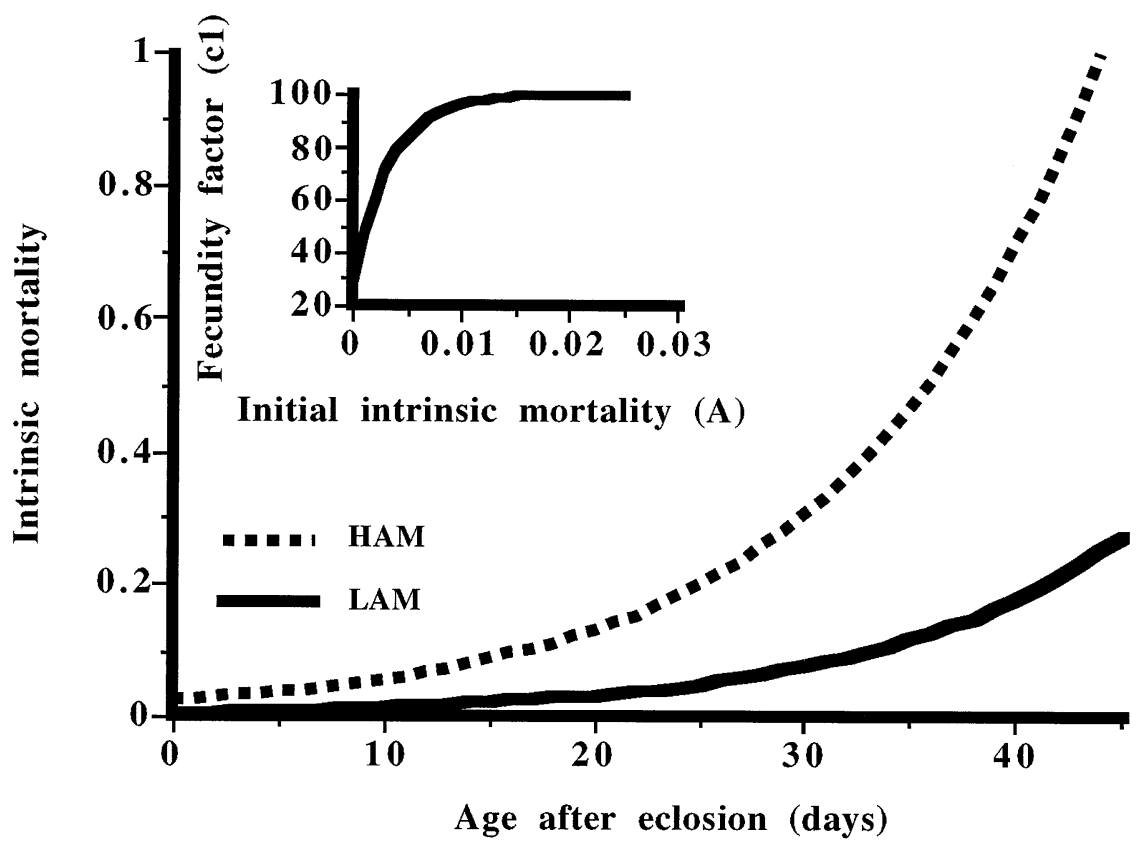

FIG. 3. Theoretical prediction of intrinsic mortality rates based on two assumptions: the intrinsic mortality has the form of a Gompertz function, and the relation between reproduction and intrinsic mortality has the form given by the inset graph. Intinsic mortality rates should be higher in HAM than in LAM flies, and the difference should increase with age.

\section{RESULTS}

Each year we measure the evolutionary changes; this takes about three months, and is done parallel to the selection experiments. Here we report the results from the 1996 measurements and partial results from the 1997 measurements, which are still being analyzed. In 1996, after three years of experimental evolution (about 75 generations in the HAM lines, about 40 generations in the LAM lines), the HAM females eclosed $17 \mathrm{~h}$ earlier, laid 10 eggs more per day early in life (aged 13-15 days from the egg) and seven eggs less per day late in life (aged 28-30 days) (Fig. 3), and had a life span that was four days shorter than the LAM females (all differences significant in a nested ANOVA). In 1996 the HAM flies also had significantly higher mortality rates in midlife (aged 24-33 days) than did the LAM flies (Fig.4); the experiment was not powerful enough to discriminate differences in morality rates later in life. Mortality rates from day 17 to day 33 were positively correlated across all replicates of both treatments with fecundity from day 13 to day 15; flies that laid more eggs early in life were more likely to die over the next two weeks than were flies that lay fewer eggs.

The comparison of the 1996 results with a partial analysis of the 1997 results suggested that all lines were moving towards earlier eclosion, smaller body size, higher early fecundity, and lower late fecundity, while HAM and LAM maintained the differences in those traits that had been established by 1996. This suggests that the trade-offs themselves are evolving; all the flies are now able to produce more eggs at a smaller body size and are able to maintain early fecundity while enclosing earlier (Fig. 5). 

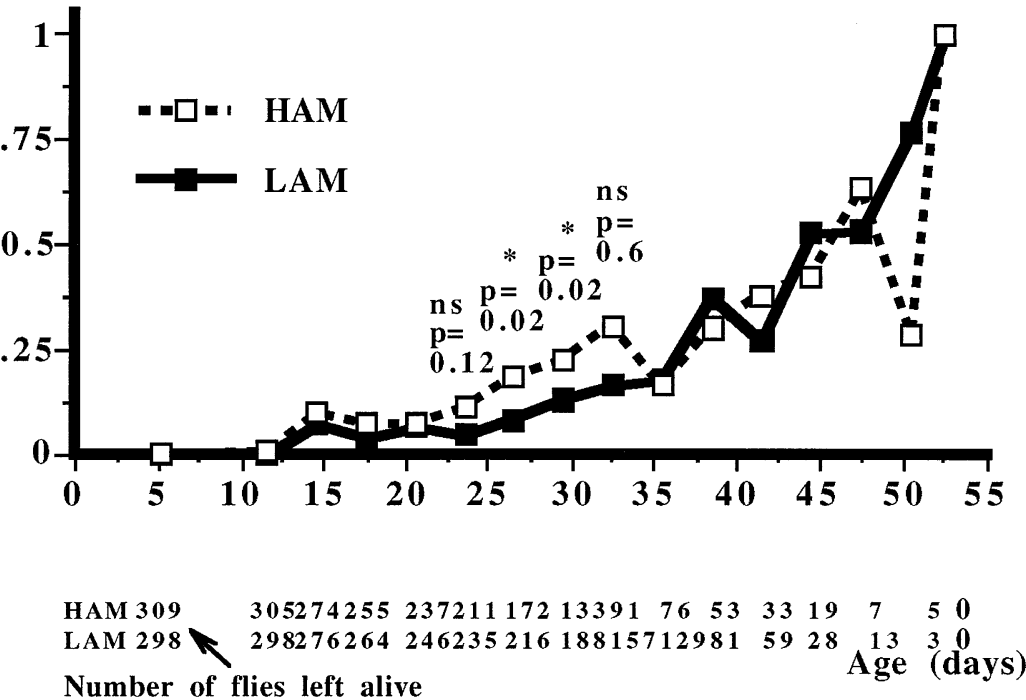

FIG. 4. The actual mortality rates observed in 1996 after 2.5 years of experimental evolution. Between 28 and 33 days of age, mortality rates were significantly higher in HAM flies than in LAM flies. Later in life sample sizes became too small to allow accurate estimates of mortality rates.

\section{DISCUSSION}

This project is the first clear experimental demonstration that intrinsic mortality rates evolve as expected in response to extrinsic mortality rates; i.e., higher extrinsic mortality rates lead to the evolution of higher intrinsic mortality rates and shorter intrinsic life spans. Thus, a central prediction of the evolutionary theory of aging has been given experimental validation.

Both the assumption that reproduction trades off with survival and the predictions of life history theory were also confirmed in an experiment where mortality rates differed but adult and larval densities did not. Increased adult mortality led to earlier maturation, smaller size at maturity, higher fecundity early in life, and lower fecundity later in life. This extends and makes more precise Reznick's results (Reznick et al., 1990) on guppies and suggests, moreover, that the interaction of extrinsic mortality rates with intrinsic trade-offs was sufficient to explain life history evolution in this experiment.

This experiment has shown that trade-offs themselves evolve as rapidly as the traits involved in the trade-offs; there has been compensatory evolution. The relationship of early fecundity to body size has been evolving rapidly in the direction of reduced costs. This confirms, in fruitflies, an important evolutionary principle already established in bacteria: that one cannot assume that because a trait is costly that it will remain costly. As soon as a shift in selection pressures makes a cost more important, evolution will act to reduce that cost.

Evolutionary biology is commonly viewed as being retrospective and descriptive. I hope that this project, and others like it, can provide experimental results to demonstrate that important aspects of evolutionary biology are theory-driven and experimental. 


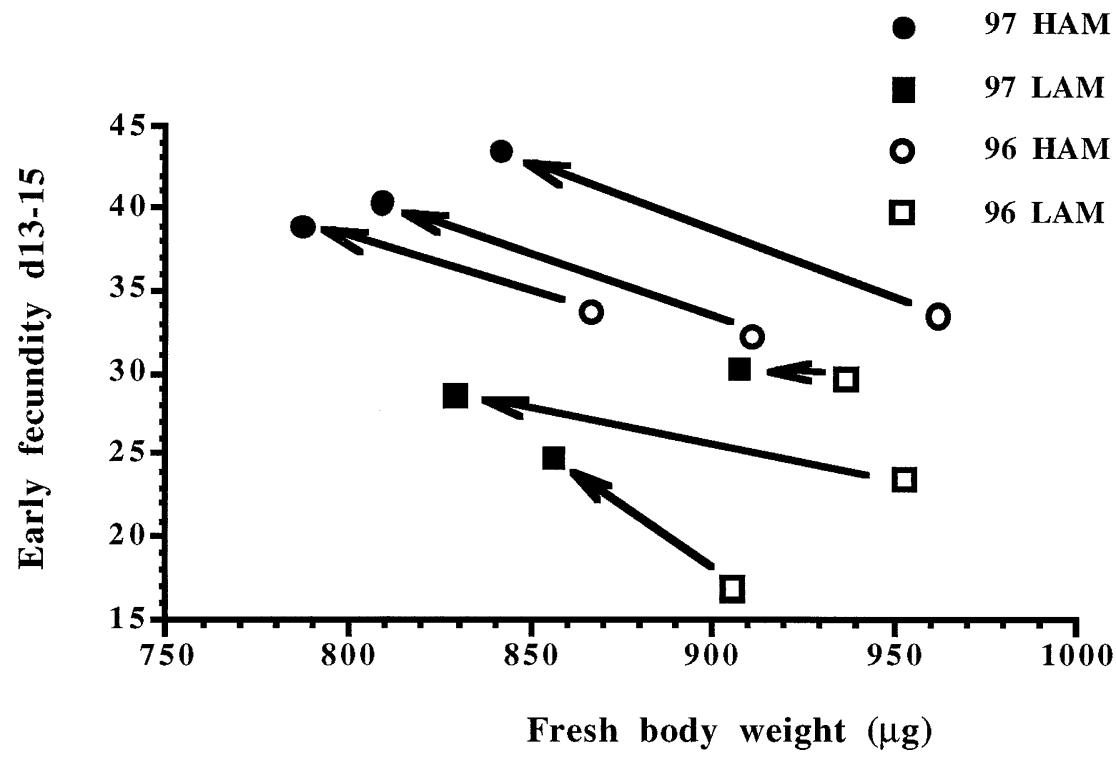

FIG. 5. A comparison of the 1996 and 1997 results for the relationship between body weight and fecundity on the 13th through 15th day of life indicates that all flies, regardless of mortality regime, are evolving smaller size and greater reproductive efficiency. In other words, one of the two key relations on which predictions were based, the relationship between body size and fecundity, is itself evolving in a way that reduces the fitness cost of being small.

Acknowledgments - I wish to thank Albert Blarer, who helped with the theoretical predictions, and Marcel Kaiser, Anni Mislin, Barbara Sykes, and Hanni Zingerli, who helped with the experiments. This research is supported by the Swiss National Science Foundation.

\section{REFERENCES}

CHARLESWORTH, B. Evolution in Age-Structured Populations, Cambridge Univ. Press, Cambridge, 1980. HAMILTON, W.D. The moulding of senescence by natural selection. J. Theor. Biol. 12, 12-45, 1966.

KIRKWOOD, T.B.L. Comparative and evolutionary aspects of longevity. In: Finch, C.E. and Schneider, E.L. (Editors), pp. 27-44, Handbook of the Biology of Aging, 2nd ed., Van Nostrand Reinhold, New York, 1985. MEDAWAR, P.B. An Unsolved Problem of Biology, H.K. Lewis, London, 1952.

PARTRIDGE, L. and BARTON, N. Optimality, mutation, and the evolution of aging. Nature 362, 305-311, 1993. REZNICK, D., BRYGA H., and ENDLER, J.A. Experimentally induced life-history evolution in a natural population. Nature 346, 357-359, 1990.

ROFF, D.A. The evolution of life histories, Chapman and Hall, London, 1992.

ROSE, M. The Evolution of Aging, Oxford University Press, New York, 1991.

STEARNS, S.C. The Evolution of Life Histories, Oxford University Press, New York, 1992.

WILLIAMS, G.C. Pleiotropy, natural selection, and the evolution of senescence. Evolution 11, 398-411, 1957. 